\title{
ClariSense+: An Enhanced Traffic Anomaly Explanation Service Using Social Network Feeds
}

\author{
Prasanna Giridhar ${ }^{\mathrm{a}}$, Md Tanvir Amin ${ }^{\mathrm{a}}$, Tarek Abdelzaher ${ }^{\mathrm{a}}$, Dong Wang ${ }^{\mathrm{b}}$, Lance \\ Kaplan $^{\mathrm{c}}$, Jemin George ${ }^{\mathrm{c}}$, Raghu Ganti ${ }^{\mathrm{d}}$ \\ ${ }^{a}$ Department of Computer Science, University of Illinois at Urbana-Champaign, Urbana, Illinois 61801 \\ ${ }^{b}$ Department of Computer Science, University of Notre Dame, Notre Dame, Indiana 46556 \\ ${ }^{c}$ Networked Sensing E Fusion Branch, US Army Research Laboratory, Adelphi, MD 20783 \\ ${ }^{d}$ IBM Research, Yorktown Heights, NY
}

\begin{abstract}
The explosive growth in social networks that publish real-time content begs the question of whether their feeds can complement traditional sensors to achieve augmented sensing capabilities. One such capability is to explain anomalous sensor readings. In our previous conference paper, we built an automated anomaly clarification service, called ClariSense, with the ability to explain sensor anomalies using social network feeds (from Twitter). In this extended work, we present an enhanced anomaly explanation system that augments our base algorithm by considering both (i) the credibility of social feeds and (ii) the spatial locality of detected anomalies. The work is geared specifically for describing small-footprint anomalies, such as vehicular traffic accidents. The original system used information gain to select more informative microblog items to explain physical sensor anomalies. In this paper, we show that significant improvements are achieved in our ability to explain small-footprint anomalies by accounting for information credibility and further discriminating among highinformation-gain items according to the size of their spatial footprint. Hence, items that lack sufficient corroboration and items whose spatial footprint in the blogosphere is not specific to the approximate location of the physical anomaly receive less consideration. We briefly demonstrate the workings of such a system by considering a variety of realworld anomalous events, and comparing their causes, as identified by ClariSense+, to ground truth for validation. A more systematic evaluation of this work is done using vehicular traffic anomalies. Specifically, we consider real-time traffic flow feeds shared by the California traffic system. When flow anomalies are detected, our system automatically diagnoses their root cause by correlating the anomaly with feeds on Twitter. For evaluation purposes, the identified cause is then retroactively compared to official traffic and incident reports that we take as ground truth. Results show a great correspondence between our automatically selected explanations and ground-truth data.
\end{abstract}

Email addresses: giridha2@illinois .edu (Prasanna Giridhar), maamin2@illinois .edu (Md Tanvir Amin), zaher@illinois .edu (Tarek Abdelzaher), dwang5@nd.edu (Dong Wang), lance.m.kaplan@us.army.mil (Lance Kaplan), jemin.george.civ@mail.mil (Jemin George), rganti@us. ibm. com (Raghu Ganti) 
Keywords: Anomaly detection, Sensors, Social data, Information gain, Credibility

\section{Introduction}

The proliferation of sensors used in human spaces, such as smart power meters, pollution meters, GPS devices, and vehicular traffic flow sensors, suggests that phenomena measured by such sensors will often be observed and reported socially as well. This is especially true of phenomena that deviate from the norm, hence attracting human attention. For example, the use of a mall's parking lot by freight trucks that increase local pollution, the closure of a freeway due to a forest fire, or the change in building occupancy patterns due to shutdown of a major local employer are events that leave a signature on both local sensors and social media. This leads to the idea of developing a service that explains anomalies seen by sensors using data feeds from social networks (e.g., Twitter [1]).

In this paper, we develop such an anomaly explanation service, with a focus on explaining small-footprint anomalies, defined as those that are localized in time and in space. We specifically apply this service to explain vehicular traffic flow abnormalities. The general working principle of such a service is conceptually simple. Given a sensor network, such as the network of traffic flow meters on city highways, and a social network, such as Twitter, the service (i) detects anomalies in sensor reports, (ii) detects anomalies in rates of different keywords in microblog items posted on the social network, (iii) filters out anomalous microblog items that lack sufficient corroboration, (iv) filters out anomalous microblog items, whose spatial signature in the blogosphere significantly exceeds that of the physical anomaly, and then finally (v) sorts the remaining set of microblog items by information gain and matches them to the set of sensors where anomalous readings are detected. A match occurs between a microblog item and a sensor if the microblog item contains keywords that refer to geographic landmarks relevant to the location of the sensor. Our results based on the traffic study show that the first one or two matching items, when considered in decreasing order of information gain, explain the sensor anomaly in most cases. Hence, a client of the service can see a map of anomaly locations (from the sensor network) and their automatically identified explanations (from the social network) that aim to clarify corresponding potential root causes. This paper describes the design and implementation of this service.

We first showcase the workings of this service for a few major real world events that caused deviation in sensor data readings. Twitter feeds corresponding to these event domains were collected and analyzed for possible explanations giving a proof that such a service is capable of identifying physical sensor anomalies with causes in any kind of settings. We then describe a case study of using this service to explain anomalies in vehicular traffic flows. Specifically, we consider real-time data feeds from traffic flow sensors in three major cities in California: Los Angeles, San Francisco, and San Diego. A public database containing this information is available at: http://pems.dot.ca.gov. The same database contains a significant number of traffic events (or incidents), such as vehicular accidents, reported road hazards, and scheduled road maintenance events, together with corresponding sensor locations and event durations. 
Real-time traffic data were collected for 3 weeks at a period of one sample (per sensor) every 5 minutes. Simultaneously, Twitter feeds were collected and ranked based on inclusion of "anomalous" keywords (i.e., those that occurred with disproportionately different frequencies before and after the sensor anomaly). Anomalous sensor readings were then matched to anomalous tweets, as described in the paper, offering clarifications of possible causes of the anomaly. A comparison of these explanations to manually collected ground truth from the incidents database suggests that our system is very good at explaining truly unusual events. The more unusual the event, the better the chances that our service automatically explains it.

It is important at this point to make a disclaimer that recognizes a limitation of this work. Specifically, it should be noted that there is an inherent conflict between demonstrating the value of our service and being able to evaluate its accuracy in a systematic manner. To evaluate accuracy systematically on a sufficiently large data set, we need to consider a case study that features anomalies for which ground truth explanations are readily available. However, the availability of ground truth explanations in such a case study is precisely what makes this case study a poor demonstration of value of the service. Said differently, since we can access police reports to understand why a traffic anomaly happenned, why do we need ClariSense+? In this paper, we opted for the systematic evaluation. Consequently, while our contribution claims lie in developing an analytical framework and demonstrating its ability to explain traffic anomalies, the purpose of publishing it is to encourage subsequent development of other applications in domains where ground truth is not as easy to come by. Various military and disaster response scenarios come to mind, but of course until the system is tested in these scenarios as well, no general applicability claims can be made beyond the current evaluation domain.

With that disclaimer in mind, the remainder of this paper is organized as follows. We describe a brief feasibility argument in Section 2. We present the system design in Section 3 . The evaluation of our system is discussed in Section 4 , followed by related work in Section 5 . In Section 6 we discuss about the things we learned from this work. Finally, conclusions are presented in Section 7

\section{A Feasibility Argument}

On June 9th, 2013, an anomalous 10-mile traffic jam was detected on a major Southern California freeway. To explain it, we contrasted two Twitter feeds; Namely, (i) Twitter feeds with keywords "California", "Traffic", and "Jam" shortly after the anomaly and (ii) Twitter feeds with the same keywords from one week earlier. The comparison returned the words "Impeach" and "Obama" as discriminative (in that they occur very frequently in the current feed while being absent from the earlier one). Picking the most common tweets containing these keywords, we obtained the following actual tweets:

@ OccupyOakland: "Impeach Obama" Gathering In California Causes 10 Mile Traffic Jam

@ justpipertoo: Impeach Obama Rally Causes 10 Mile Traffic Jam on a Southern California overpass 
Indeed, it turned out that the traffic jam was caused by a rally organized by the Tea Party. The above example clearly demonstrates the utility of exploiting correlated anomaly detection in social and sensor networks for explaining the causes of anomalies seen by sensors. While the above was done manually, the incident suggests that a general sensor anomaly explanation service can be developed that aims to automatically clarify root causes of sensor anomalies. In this paper, we focus on explaining small-footprint anomalies such as local traffic flow disruptions. The architecture, im-

plementation, and evaluation of such an anomaly explanation service are the topic of this paper.

\section{System Design}

The general architecture of our service is shown in Fig 1 . As the figure suggests, several challenges need to be resolved for the service to work properly. We first describe a generic framework, giving an outline of each of these challenges, and then we present a detailed study focusing on a real-world scenario (i.e., the road traffic network).

We initialize the system with social feeds and data from physical sensors. The key property we need for the mechanisms in this paper to work well is that the sensors measure physical quantities that result from events that are also witnessed by people who are willing to share their observations via social media. For such events, we focus on automating the scanning of social media to find related social feeds in time and space that might explain the anomalous sensor readings. Sensor anomaly detection algorithms have been discussed at length in prior literature and thus are not investigated in this work.

Our algorithm determines the most discriminative microblog items from a given set of social feeds. It does so by identifying anomalous keyword combinations in today's social feeds (that may characterize different events). The problem can be thought of as one of classification. Given a set of bins with today's microblog items and a set of bins with past microblog items, which keyword combinations (from these microblogs) offer the maximum information gain in distinguishing the two types of bins? The candidate keyword combinations to be considered are chosen by looking at words that co-occur in at least one microblog entry. We also remove retweets before finding the keyword combinations. This step is important since retweets do not add independent information and hence should not increase information gain.

The next task after the identification of discriminative word combinations is to identify all the microblog items that contain at least one of these discriminative word combinations. Such microblog items are deemed anomalous and are candidates for explaining today's sensor anomalies. Some of these lack sufficient corroboration and are thus removed. Of the remaining ones, there might be discriminative (i.e., highinformation-gain) highly corroborated items that are not related to the local sensor anomalies. For example, today's national breaking news might be discussed in the blogosphere and appear today with a high degree of corroboration, but not earlier. These items will thus be discriminative when feeds from a given location are collected, but they might in fact have no relation to events at that particular location. This is a 
specificity problem; such feeds are not specific to the location of interest. The following are examples of such tweets for the query term "traffic":

- Yahoo topped Google in US traffic in July http://t.co/o3v0rdYqYh

- Google Brings Traffic Updates To Maps App; Adds Search to Waze App by @ mattsouthern: Google announced this morning http://t.co/vR2bbPQEDf

- NSA surveillance covers 75 percent of U.S. Internet traffic: WSJ: NEW YORK (Reuters) http://t.co/kYuMJUEhEM

It can be seen that these tweets are not about local "traffic". Rather, they refer to breaking news about Google, Yahoo, and the NSA. Such tweets are not location specific and will tend to be discriminative at many different locations. We exploit this insight by adding a spatial locality analysis technique to give preference to tweets that are location-specific.

It remains to match the surviving anomalous microblog entries to the correct physical sensor anomaly they might explain. This is done by matching the social and physical anomalies in space and in time. In other words, the anomalous microblog entries must occur shortly after or around the sensor anomaly and must contain keywords that one might use when referring to the location of the sensor. For the purposes of location matching, we associate each physical sensor with a set of keywords describing local landmarks that might be mentioned in a microblog when referring to the corresponding location. We take the advantage of Google Maps feature using the geo-coordinates of the sensor location to find these keywords. During our case study on traffic data each physical sensor had metadata providing the coordinate information, thereby making the process automated. Location matching between sensor anomalies and social anomalies is then done by matching keywords that occur in the anomalous microblog entry to keywords associated with different sensor locations, where physical anomalies were observed. Once matching is complete, the results are displayed on a map, highlighting locations of sensor anomalies and the candidate explanations matched to each in corresponding text bubbles.

One important observation is that all computations described above are done without understanding or interpreting microblog text. Information gain, degree of corroboration, and spatial footprint are all computed based on statistics of occurrence of different keywords and microblog items. Semantic analysis of items is not used. While, in principle, such semantic analysis may improve results, the paper shows that our ability to explain small-footprint anomalies, such as traffic flow disruptions, is sufficiently high even using the statistical techniques only without understanding text semantics.

Table 1 shows examples of anomalous sensor readings matched to Twitter explanation. In this table we provide the ground truth data for each anomaly obtained manually from news and reliable online sources, physical sensor data used to detect these anomalies, and the identified tweet explanations by our service that match the ground truth.

In the following subsections, we provide a detailed overview of the system applied to explain anomalies in vehicular traffic data. We consider this case study for two reasons; namely, (i) traffic features more frequent anomalies within a limited timeframe than other domains, and (ii) groud truth is readily available for each observed anomaly, which enables evaluation of system accuracy. The sensor data were retrieved from 
Table 1: Sensor anomalies with identified explanations using generic datasets

\begin{tabular}{|c|c|c|}
\hline Ground Truth & Physical Sensor & Tweet Explanation \\
\hline $\begin{array}{l}\text { In May } 2015 \text { a deadly flood affected } \\
\text { communities across the north-west Texas } \\
\text { state. The National Weather Service } \\
\text { offices in Texas issued } 594 \text { flash flood } \\
\text { warnings which was on average more } \\
\text { than the previous year. }\end{array}$ & $\begin{array}{l}\text { Harris County flood warning system [2] } \\
\text { provides the average rainfall recieved per } \\
\text { day. There were spikes in the data for the } \\
\text { last week of May indicating an anomaly. }\end{array}$ & $\begin{array}{l}\text { (1) UPDATE: Disaster Declaration for } \\
\text { historic Texas flooding now includes } 70 \\
\text { counties \#txwx \#hounews } \\
\text { (2) } 70 \text { out of Texas' } 254 \text { counties are } \\
\text { *currently* on the list for disaster decla- } \\
\text { rations. More could be added. \#txwx } \\
\text { (3) Gov. Abbott requests Presidential } \\
\text { disaster declaration for Texas counties } \\
\text { http://t.co/u8NvhMQKgD } \\
\text { (4) President Obama Declares Ma- } \\
\text { jor Disaster In Texas After Deadly } \\
\text { Floods http://t.co/OyUODHeKpr } \\
\text { http://t.co/hx8tU3fFBh }\end{array}$ \\
\hline $\begin{array}{l}\text { The } 2015 \text { April Nepal earthquake killed } \\
\text { more than } 9,000 \text { people and injured more } \\
\text { than } 23,000 \text { with a magnitude of } 7.8 \mathrm{M} \text {. It } \\
\text { was the worst natural disaster to strike } \\
\text { Nepal since the } 1934 \text {. }\end{array}$ & $\begin{array}{l}\text { National Seismological Centre, Nepal } \\
{[3 \text { measures the magnitudes of seismic }} \\
\text { waves using physical sensing devices } \\
\text { and provides the data publicly. The ob- } \\
\text { servations for last week of April indicate } \\
\text { an anomalous event activity. }\end{array}$ & 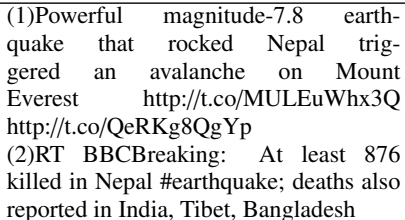 \\
\hline
\end{tabular}

the Caltrans Performance Measurement System (PeMS) [4]. This system provides data corresponding to flow values, average speed, occupancy and average delay for all major freeways in California, USA. The physical sensors on the freeways record values every 5 minutes. The experiment was conducted for a period of 3 weeks starting from July 14, 2014, to August 3, 2014, during which all major incidents were collected from 3 cities, namely, Los Angeles, San Diego and San Francisco. We collected tweets from the aforementioned 3 cities using traffic as the keyword.

A separate collection window was used for training data in order to set the various algorithm parameters. Training data were collected for the same three cities from California mentioned above for a period of two weeks starting from August 19, 2013, to September 1, 2013, which again had values corresponding to flow, average speed, occupancy and average delay for all major freeways at an interval of 5 minutes.

\subsection{Identifying Sensor Anomalies}

The first challenge was to determine what constitutes a sensor anomaly. In many cases, the definition of anomalies is context sensitive. For example, what constitutes anomalous traffic at midnight might be perfectly normal at rush hour. For the purposes of evaluation in this paper, we considered flow anomalies that were observed on major freeways in the California road traffic network. The different anomaly types can be classified as Accidents involving vehicle collisions or hit and run cases, Hazards involving interruption due to blockage by some object such as tree, Breakdowns involving car fire or failure, Weather-related traffic incidents, and Other events (e.g., road construction).

In the current evaluation, a sensor anomaly is determined by extracting the California Highway Patrol incidents using the Performance Management Systems (PEMS) analysis tool on all major freeways (in our target cities), which reports the start, end, duration, and sensor IDs for each detected anomaly. In order to remove redundant 


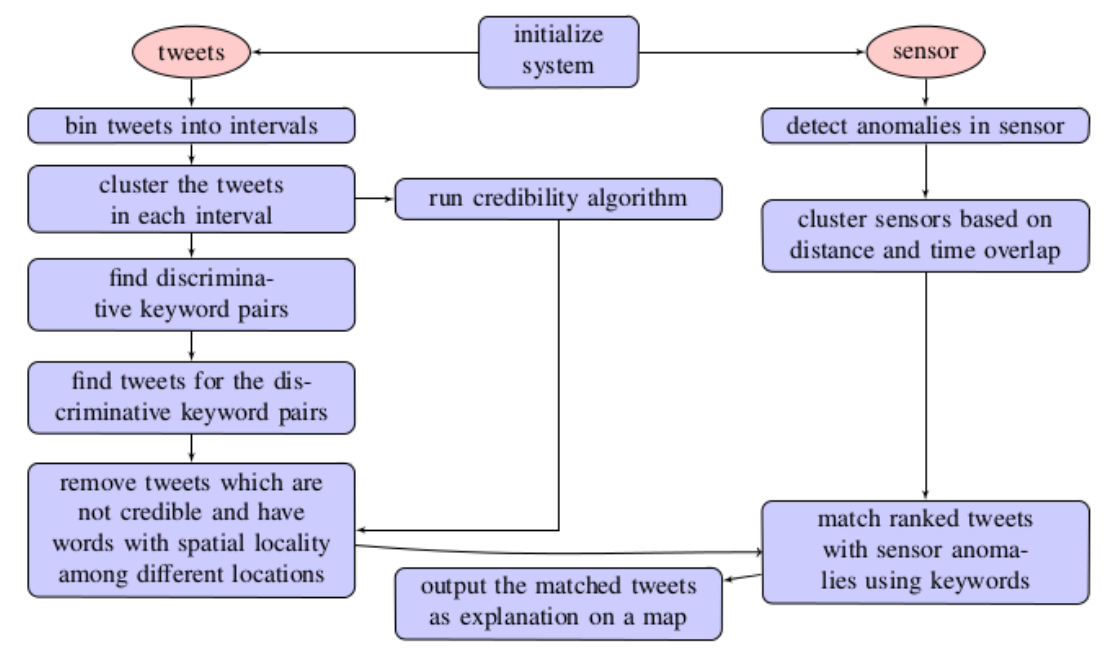

Figure 1: ClariSense+ Architectire

observations of the same anomaly, we perform clustering of nearby sensor anomaly reports, since such clusters are typically triggered by the same physical event.

To determine the condition for considering two sensor anomaly reports to be in the same cluster, we identified anomalous traffic events that co-occur on a given day around the same time and computed the distance distribution between them. This is the distribution of inter-cluster distances. We then selected a much smaller number (namely, two miles) to indicate membership in the same cluster. Hence, for clustering purposes, we consider anomalous sensor readings that are less than 2 miles apart to be part of the same event cluster. A single event explanation is assigned to each cluster. This is in line with our focus on small-footprint events (as opposed to, say, wars, hurricanes, and earthquakes that may affect areas that are hundreds of miles wide).

\subsubsection{Identifying Events}

In order to identify anomalous events described in social network feeds, one must first decide what constitutes a distinct events. A first instinct might be to do some semantic analysis of tweets to recognize distinct events. The approach requires detailed language models and hence is hard to apply in situations where informal abbreviations, hashtags, and slang are commonly used. Instead, we opted for the minimalist approach. We hypothesized that when individuals describe the same event, they use event-related keywords. The problem is that the relation between individual events and individual keywords might not be one-to-one. Hence, one cannot uniquely associate individual keywords with individual events. The question is, can one automatically find a set of keywords that would have a one-to-one correspondence with a unique event (to use as an event signature)?

To answer the above question, we first removed all words that are less than fourletters long, then used a part-of-speech tagger to identify (English) nouns. These nouns 
Table 2: Event Signatures

\begin{tabular}{|c|c|c|}
\hline Signature & Events per Signature & Signatures per Event \\
\hline \hline Single Keyword & 3.621 & 1.1579 \\
\hline Keyword Pair & 1.1416 & 1.2725 \\
\hline Keyword Triplet & 1.0628 & 0.4393 \\
\hline
\end{tabular}

served as keywords. Candidate event signatures were then automatically found by identifying keyword combinations with high information gain. Three types of candidate event signatures were compared; namely, signatures that comprised a single keyword (e.g., "fire"), those that comprised a keyword pair (e.g., "fire", "stadium"), and those that comprised a keyword triplet (e.g., "fire", "stadium", "deaths"). To do so, all possible combinations of keywords (nouns of four letters or more) were first extracted from each tweet, then the resulting list of keyword combinations was ranked by information gain. Each ranked combination of keywords must have therefore co-occurred in at least one tweet, not necessarily contiguously. Combinations whose information gain exceeded a threshold were deemed discrinimative, meaning that these combinations occurred disproportionally more frequently within today's tweets compared to normal. We manually read all discriminative combinations and manually grouped them by the event they describe. Informally, an event was defined to be a distinct traffic incident.

The results were used to answer two questions: For each signature category, (i) how many distinct physical events match a single signature, and (ii) how many distinct signatures match a single physical event? Ideally, both answers should be close to 1 for a one-to-one mapping between events and their signatures. This would allow us to find discriminative events by looking for discriminative signatures. Note that, if an anomalous event matches more than one signature, it may be that no single signature is discriminate and hence we fail to identify this event. Conversely, if many events match the same signature, anomalous variations in the number of references to individual events might not translate to anomalous variations in the number of occurrences of this signature (e.g., they may cancel out). Thus, the anomalous events may again be missed when looking at variations in occurrence of the signatures. In short, one needs a close to one-to-one mapping between signatures and events in order for anomalous counts of event references to translate into anomalous signature counts. Table 2 shows our results for the three types of signatures considered for the events identified from our training data set. It can be seen that keyword pairs come closest to a one-to-one correspondence between independent events and their keyword signatures. We therefore adopt a twoword signature in the rest of the paper to identify distinct events. Hence, to explain anomalous events, we need to detect anomalous keyword pairs then match them to the right anomalous sensor report cluster. Below, we describe how anomalous keyword pairs are selected.

\subsubsection{Ranking Signatures by Information Gain}

Our goal is to identify keyword pairs that describe anomalous events. To do so, we seek keyword pairs that occur disproportionately frequently at the current time, compared to their average frequency in the past. The "current time" is defined as a 


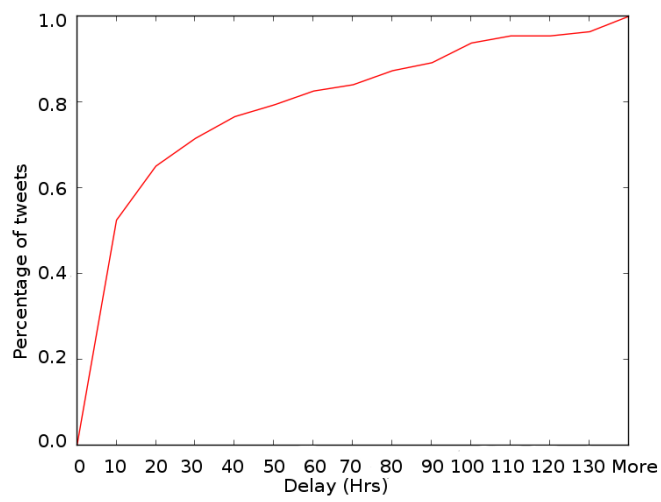

Figure 2: Distribution of retweet delays

sufficiently large window, called the current window, during which most tweets of the same physical event will likely arrive. For small-footprint events, such as traffic accidents, unusual traffic jams, fires, and closures, we empirically computed this window by obtaining the distribution of retweet delays (time difference between each retweet and the original tweet). We plotted a cumulative delay distribution for retweets in our training dataset, as shown in Fig 2 where the horizontal axis represents the delay within which the indicate percentage of retweets occur. The figure shows that around $70 \%$ of retweets occur within 24 hours of the original traffic event. Hence, we break the Twitter data feed into fixed windows that are 24 hours each.

The simplest way to identify disproportionately frequent event references in the current window is to compute their rate difference from normal. In other words, compare the rate of these references within the current window to their average rate in the past window. The problem with simply computing the rate difference is that it may favor high volume to disproportionality. For example, a keyword pair such as (traffic, jam) that appears 50 times today, versus an average of 35 times a day, will have a larger rate difference compared to a pair such as (drunk, kills) that appears 12 times today compared to a past average of 0 . Intuitively, the latter is a more anomalous event.

The above might suggest that we take the ratio of current to average rates, as opposed to the difference. This approach has its own problems. For example, a keyword pair that appears once today but never before will have a larger current-to-average rate ratio than a pair that appears 50 times today, but only once on average. Intuitively, the latter is more important. The former could be an entirely random occurrence (such as a typo in one of today's tweets). An approach that is not biased by the normal frequency of the pairs is desired.

One such approach, which has the advantage of having well-understood analytic semantics, is to use information gain. Consider the set $W$ of all word pairs that occurred in today's tweets (i.e., each was present in at least one tweet). Let $W_{i}$ denote the $i$ th word pair. Let $Y$ be a binary variable that denotes the window being considered, such that $Y=0$ in today's window and $Y=1$ in the past (normal) window. Let $X_{i}$ 
be the binary variable that denotes the absence $\left(X_{i}=0\right)$ or presence $\left(X_{i}=1\right)$ of the $i$ th word-pair in an observation window. Our goal is to find the top $K$ discriminative keyword pairs in the sense of maximizing information gain and hence better distinguishing the normal from the anomalous windows. For each keyword pair, $W_{i}$, we compute information gain, $I G_{i}$ from the standard definition:

$$
I G_{i}=H(Y)-H\left(Y \mid X_{i}\right)
$$

where $H(Y)$ is the entropy associated with variable $Y$ and $H\left(Y \mid X_{i}\right)$ is the conditional entropy of the variable $Y$ given $X_{i}$ (i.e., given the presence or absence of the $i$ th word pair). Note that, the keyword pair that maximally reduces conditional entropy, maximizes information gain. The following expressions define the entropy computations:

$$
\begin{aligned}
H(Y) & =-\sum_{y \in Y} p(y) \log _{2} p(y) \\
H\left(Y \mid X_{i}\right) & =\sum_{x \in X_{i}} p(x) H\left(Y \mid X_{i}=x\right) \\
& =-\sum_{x \in X_{i}} p(x) \sum_{y \in Y} p(y \mid x) \log _{2} p(y \mid x) \\
& =-\sum_{x \in X_{i}} \sum_{y \in Y} p(x, y) \log _{2} p(y \mid x)
\end{aligned}
$$

Note that, since the entropy of $Y$ is constant for all keywords, we need to evaluate only the conditional entropy of $Y$ given $X_{i}$. The lower the value of conditional entropy the more discriminative the given word pair is. Hence, the algorithm finds the top $K$ discriminative keyword pairs in the sense of minimizing conditional entropy.

The algorithm simply computes the equation (2) to find the conditional entropy associated with a word pair. The probability values indicated in the equation are calculated as follows:

$$
\begin{aligned}
\log _{2} p(y \mid x) & =\log _{2} p(x, y)-\log _{2} p(x) \\
p(x, y) & \approx \frac{\operatorname{count}(X=x, Y=y)}{\sum_{x \in X, y \in Y} \operatorname{count}(X=x, Y=y)} \\
p(x) & \approx \frac{\operatorname{count}(X=x)}{\sum_{x \in X} \operatorname{count}(X)}
\end{aligned}
$$

In equation (3) we use the count of each pair associated with an event type to determine the probability of observing the event in its presence and absence. We also determine the fraction in which the word pair is present or absent. Thus, using these values, we calculate the discriminative factor associated with a pair in the current window in comparison to the previous window.

Once the most discriminative keyword pairs are found, we find tweets that contain these pairs. These are the discriminative tweets. We rank them by conditional entropy 
Table 3: Top 10 tweets from San Diego with high Information Gain

\begin{tabular}{|c|l|c|}
\hline Rank & \multicolumn{1}{|c|}{ Tweets } & Keyword Pair \\
\hline \hline 1 & $\begin{array}{l}\text { Google Brings Traffic Updates To Maps App; Adds Search to Waze App by } \\
\text { @ mattsouthern: Google announced this mor... http://t.co/vR2bbPQEDf }\end{array}$ & (search, google) \\
\hline 2 & $\begin{array}{l}\text { I like this Google Adds Waze Traffic To Maps App, Search To Waze \#seo } \\
\text { http://t.co/kttjnBTXIU }\end{array}$ & (search, google) \\
\hline 3 & $\begin{array}{l}\text { \#SEO -\&gt; Google Adds Waze Traffic To Maps App, Search To Waze } \\
\text { http://t.co/W8mcY5aBVe }\end{array}$ & (search, google) \\
\hline 4 & $\begin{array}{l}\text { Next iteration of local \#mobile as \#Google Adds \#Waze Traffic To Maps } \\
\text { App, Search To Waze http://t.co/ehVCg71cSh \#localsearch \#mobileseo }\end{array}$ & (search, google) \\
\hline 5 & $\begin{array}{l}\text { WSJ reports NSA spying capabilities cover up to 75 percent of US internet } \\
\text { traffic http://t.co/DQpLj3ciQr }\end{array}$ & (internet, percent) \\
\hline 6 & $\begin{array}{l}\text { NSA surveillance covers 75 percent of U.S. Internet traffic: WSJ: NEW } \\
\text { YORK (Reuters) - The National Security A... http://t.co/kYuMJUEhEM }\end{array}$ & (internet, percent) \\
\hline 7 & $\begin{array}{l}\text { NSA can reach 75 percent of US internet traffic, reports WSJ } \\
\text { http://t.co/xDFR8iDVe1 }\end{array}$ & (internet, percent) \\
\hline 8 & $\begin{array}{l}\text { TRAFFIC ALERT: At least 1 person hurt after crash with Sycuan bus. } \\
\text { Dehesa Road is closed. http://t.co/iXdRCffFlG http://t.co/9FhmKl2BGd }\end{array}$ & (alert, dehesa) \\
\hline 9 & $\begin{array}{l}\text { Traffic Alert: Casino bus crashes with car - Dehesa Rd. closed } \\
\text { http://t.co/eQ9UCMF8D8 }\end{array}$ & (alert, dehesa) \\
\hline 10 & $\begin{array}{l}\text { Culture Report: Foot Traffic Way Up at One Balboa Park Museum } \\
\text { http://t.co/Fn9d9nQG6r }\end{array}$ \\
\hline
\end{tabular}

such that tweets containing more discriminative pairs come first. These tweets constitute candidate explanations of observed sensor anomalies. Table 3 shows the top 10 results for San Diego city ranked according to the derived information gain equation, and computed over the window belonging to August 21, 2013 contrasted against the previous day feeds.

Since some high-information-gain items might contain false information, we next describe an approach that incorporates credibility into calculations of information gain.

\subsubsection{Filtering tweets with credibility information}

After finding the information gain value for each of the discriminative tweets (i.e., tweets containing discriminative keyword pairs), we further include another variable $Z$, which is the vector associated with the latent truth values of the microblog items in question. Each element in this vector can take two possible values, 1 indicating that the corresponding item contains a true claim and 0 indicating that the claim is false. Past work [5] describes an approach to find the maximum likelihood truth value associated with a claim based on a graph formed between sources and claims. It iteratively converges to these values using an expectation maximization algorithm. The credibility scores are indicated in the form of probability values, which can be easily accounted for in the information gain equation by making the observation that falsehoods offer zero information on the actual physical state of the sensors. Therefore, regardless of how discriminative a keyword pair is, if the microblog item containing it is false, it does not have any correspondence to any traffic events, and thus offers no useful information on traffic events. The expression of information gain and entropy are therefore informally redefined to count true claims only. Hence, we have:

$$
I G_{i}\left(Y ; X_{i}, Z_{i}\right)=H\left(Y \mid Z_{i}\right)-H\left(Y \mid X_{i}, Z_{i}\right)
$$

where $H\left(Y \mid Z_{i}\right)$ is the entropy associated with variable $Y$, given the truth value $Z_{i}$. Since we count only true claims, the effect of $Z_{i}$ is to eliminate all false claims when com- 
puting $H\left(Y \mid Z_{i}\right)$. Similarly, $H\left(Y \mid X_{i}, Z_{i}\right)$ is the conditional entropy of the variable $Y$ given claim $X_{i}$ and the truth value $Z_{i}$. The following expressions define the modified entropy and information gain:

$$
\begin{aligned}
H\left(Y \mid Z_{i}\right) & =\sum_{z \in Z_{i}} p\left(Z_{i}=z\right) H\left(Y \mid Z_{i}=z\right) \\
H\left(Y \mid X_{i}, Z_{i}\right) & =\sum_{z \in Z_{i}} p\left(Z_{i}=z\right) H\left(Y \mid X_{i}, Z_{i}=z\right) \\
I G_{i}\left(Y ; X_{i}, Z_{i}\right) & =\sum_{z \in Z_{i}} p\left(Z_{i}=z\right)\left[H\left(Y \mid Z_{i}\right)-H\left(Y \mid X_{i}, Z_{i}\right)\right] \\
& =p\left(Z_{i}=1\right)\left[H\left(Y \mid Z_{i}=1\right)-H\left(Y \mid X_{i}, Z_{i}=1\right)\right] \\
& +p\left(Z_{i}=0\right)\left[H\left(Y \mid Z_{i}=0\right)-H\left(Y \mid X_{i}, Z_{i}=0\right)\right]
\end{aligned}
$$

In the last equation above, note that since we redefined entropy and information gain to count true claims only, the expresions for $H\left(Y \mid Z_{i}=0\right)$ and $H\left(Y \mid X_{i}, Z_{i}=0\right)$ are identical since $X_{i}$ would be filtered out from the the body of tweets (it would be removed as false) when $Z_{i}=0$. Hence, $H\left(Y \mid Z_{i}=0\right)-H\left(Y \mid X_{i}, Z_{i}=0\right)=H\left(Y \mid Z_{i}=0\right)-H\left(Y \mid Z_{i}=0\right)=0$. Also, $H\left(Y \mid Z_{i}=1\right)=H(Y)$ and $H\left(Y \mid X_{i}, Z_{i}=1\right)=H\left(Y \mid X_{i}\right)$, since no tweets are filtered out on account of being false when $Z=1$. Substituting above, we get:

$$
I G_{i}\left(Y ; X_{i}, Z_{i}\right)=p\left(Z_{i}=1\right) I G_{i}
$$

where $p\left(Z_{i}=1\right)$ is computed using a previously published credibility analysis algorithm [5]. Table 4 shows the top 10 tweets ranked according to the new information gain equation derived using the credibility of the claims for the same date from San Diego city. Comparing with the results of Table 3, we find that claims have been rearranged based on their perceived credibility as computed by the maximum likelihood estimation algorithm.

As seen in Table 4 some results belong to news regarding the internet traffic generated by Google, which got picked up due to high information gain values and high credibility in that particular window during the analysis. This is a common occurrence in that national news will always have a high information gain. One should observe, however, that national news, being of global interest, will offer a high information gain in multiple locations. This offers a clue on how to identify such items and remove them when the objective is to focus on local (i.e., small-footprint) anomalies. A simple technique is described in the following section.

\subsubsection{Spatial analysis to remove spatially non-specific claims}

Global news items are generally not specific to the location of the local sensor anomaly. They offer high information gain in feeds collected from other geographic locales as well. Hence, when collecting tweets about traffic in different California cities, it so happened that some of the top discriminative and credible tweets were related to global news. For example, the acquisition of Waze by Google to offer traffic information on Google Maps was high information-gain breaking news. All the tweets related 
Table 4: Top 10 tweets from San Diego with high Information Gain and Credibility

\begin{tabular}{|c|l|c|}
\hline Rank & \multicolumn{1}{|c|}{ Tweets } & Keyword Pair \\
\hline \hline 1 & $\begin{array}{l}\text { \#SEO-\&gt; Google Adds Waze Traffic To Maps App, Search To Waze } \\
\text { http://t.co/W8mcY5aBVe }\end{array}$ & (search, google) \\
\hline 2 & $\begin{array}{l}\text { I like this Google Adds Waze Traffic To Maps App, Search To Waze \#seo } \\
\text { http://t.co/kttjnBTXIU }\end{array}$ & (search, google) \\
\hline 3 & $\begin{array}{l}\text { NSA can reach 75 percent of US internet traffic, reports WSJ } \\
\text { http://t.co/xDFR8iDVe1 }\end{array}$ & (internet, percent) \\
\hline 4 & $\begin{array}{l}\text { Report: The \#NSA can access 75\% of all U.S. internet traffic } \\
\text { http://t.co/WzDSxorWa6 }\end{array}$ & (internet, report) \\
\hline 5 & $\begin{array}{l}\text { TRAFFIC ALERT: At least 1 person hurt after crash with Sycuan bus. } \\
\text { Dehesa Road is closed. http://t.co/iXdRCfvFlG http://t.co/9FhmKl2BGd }\end{array}$ & (alert, dehesa) \\
\hline 6 & $\begin{array}{l}\text { TRAFFIC: A passenger bus collided with a car on Dehesa Rd. near } \\
\text { \#Sycuan casino: http://t.co/MqGIvS0vsU http://t.co/2cytXqMiET }\end{array}$ & (casino, dehesa) \\
\hline 7 & $\begin{array}{l}\text { Traffic Alert: Casino bus crashes with car - Dehesa Rd. closed } \\
\text { http://t.co/eQ9UCMF8D8 }\end{array}$ & (alert, dehesa) \\
\hline 8 & $\begin{array}{l}\text { Culture Report: Foot Traffic Way Up at One Balboa Park Museum } \\
\text { http://t.co/Fn9d9nQG6r }\end{array}$ & (balboa, museum) \\
\hline 9 & $\begin{array}{l}\text { WSJ reports NSA spying capabilities cover up to 75 percent of US internet } \\
\text { traffic http://t.co/DQpLj3ciQr }\end{array}$ & (balboa, museum) \\
\hline 10 & $\begin{array}{l}\text { NSA surveillance covers 75 percent of U.S. Internet traffic: WSJ: NEW } \\
\text { YORK (Reuters) - The National Security A... http://t.co/kYuMJUEhEM }\end{array}$ & (internet, percent) \\
\hline & \multicolumn{2}{|c|}{} \\
\hline
\end{tabular}

Table 5: Spatial Analysis

\begin{tabular}{|c|c|c|c|c|c|c|c|}
\hline \multicolumn{2}{|c|}{} & \multicolumn{2}{|c|}{ SF } & \multicolumn{2}{|c|}{ SD } & \multicolumn{2}{c|}{ LA } \\
\hline $\mathrm{N}$ & $\mathrm{C}$ & $\mathrm{T}$ & $\mathrm{E}$ & $\mathrm{T}$ & $\mathrm{E}$ & $\mathrm{T}$ & $\mathrm{E}$ \\
\hline All & 299 & 2 & 0 & 3 & 0 & 15 & 0 \\
\hline 500 & 113 & 14 & 0 & 15 & 0 & 112 & 2 \\
\hline 300 & 76 & 22 & 0 & 21 & 0 & 149 & 2 \\
\hline 200 & 58 & 28 & 0 & 22 & 0 & 170 & 2 \\
\hline 100 & 34 & 36 & 0 & 30 & 1 & 237 & 2 \\
\hline 50 & 23 & 46 & 1 & 38 & 1 & 282 & 3 \\
\hline 20 & 10 & 59 & 1 & 53 & 1 & 360 & 3 \\
\hline 10 & 3 & 85 & 1 & 85 & 1 & 495 & 3 \\
\hline 5 & 1 & 97 & 1 & 95 & 1 & 510 & 3 \\
\hline
\end{tabular}

to this event got ranked high for that particular window after applying the equations described above.

In order to eliminate items that are of global interest, as opposed to location-specific items, we looked at the top single keywords for each day sorted by frequency of their occurrence for each of the cities where data were collected. On looking at the top $N$ words, we discovered that most of them were common across all the cities. We considered that a keyword is likely to refer to a local event if it is exclusive and localized to the feed from a particular city. Hence, all words that are shared in the top $N$ lists were eliminated from consideration. Keyword pairs containing any of these words were removed.

The choice of $N$ offers a trade-off between false positives (items not related to local anomalies) and false negatives (actual anomalies that are not detected). A high value of $N$ will increase false negatives, whereas a small value will increase false positives. To find a good value of $N$, we collected training data set from the three cities. Table 5 
Table 6: Top 10 tweets from San Diego with high Information Gain and Spatial Analysis

\begin{tabular}{|c|l|c|}
\hline Rank & \multicolumn{1}{|c|}{ Tweets } & Keyword Pair \\
\hline \hline 1 & $\begin{array}{l}\text { TRAFFIC ALERT: At least 1 person hurt after crash with Sycuan bus. } \\
\text { Dehesa Road is closed. http://t.co/iXdRCfvFlG http://t.co/9FhmKl2BGd }\end{array}$ & (alert, dehesa) \\
\hline 2 & $\begin{array}{l}\text { Traffic Alert: Casino bus crashes with car - Dehesa Rd. closed } \\
\text { http://t.co/eQ9UCMF8D8 }\end{array}$ & (alert, dehesa) \\
\hline 3 & $\begin{array}{l}\text { Culture Report: Foot Traffic Way Up at One Balboa Park Museum } \\
\text { http://t.co/Fn9d9nQG6r }\end{array}$ & (balboa, museum) \\
\hline 4 & $\begin{array}{l}\text { Think I'll stop by the \#Timken too! RT @ voiceofsandiego Culture Report: } \\
\text { Foot Traffic Way Up at One Balboa Park Museum http://t.co/oucC1yqeKJ }\end{array}$ & (balboa, museum) \\
\hline 5 & $\begin{array}{l}\text { TRAFFIC: A passenger bus collided with a car on Dehesa Rd. near } \\
\text { \#Sycuan casino: http://t.co/MqGIvS0vsU http://t.co/2cytXqMiET }\end{array}$ & (casino, dehesa) \\
\hline 6 & $\begin{array}{l}\text { @leebrice yes please!!!! Just a short road trip up from sunny San Diego for } \\
\text { my sis and me!!! We can have a \#parkinglotparty in LA traffic! }\end{array}$ & (please, diego) \\
\hline 7 & $\begin{array}{l}\text { Vote for MAD TRAFFIC, please, and attend a San Diego tradition: } \\
\text { http://t.co/WdvCAYkngv }\end{array}$ & (please, diego) \\
\hline 8 & $\begin{array}{l}\text { TRAFFIC ALERT: Sig Alert issued for southbound I-805 at eastbound } \\
\text { SR-54 due to car stuck in No. 1 lane. }\end{array}$ \\
\hline 9 & $\begin{array}{l}\text { omg not used to a real schedule anymore. and i forgot how much i had to do } \\
\text { this am. time for a monster and traffic! weeee }\end{array}$ & (anymore, schedule) \\
\hline 10 & San Diego has been nice apart from the traffic & (apart, diego) \\
\hline
\end{tabular}

shows how the number of identified events varies as we vary the value of $N$ in the first column for the date August 21, 2013. The single keywords for each of the city were sorted in increasing order of their frequency of occurrence and we searched for the common words among all cities for the top $N$ keywords. The number of common words found is indicated in the second column (C) of the table for all values of $N$. We next removed all the tweets from the ranked credibility-information gain list which contained these common words and retained the remaining tweets to compare with physical sensor anomalies. Thus, for each of the three cities, we have two sub-columns indicating the number of tweets $(\mathrm{T})$ retained after spatial analysis and the number of identified events (E) matching with physical sensor anomalies. From the table, we can see that $N=50$ retains the maximum number of true positives. That is to say, reducing $N$ below 50 does not help identify more events, whereas increasing it results in false negatives. Therefore, the empirical value of $N$ was set as 50 for the implementation and evaluation part in this paper.

Table 6 shows the top 10 tweets with high information gain and spatial analysis from San Diego city. If we closely compare the results of this table with that of Table 4 . we can observe that all those tweets that were not related to road traffic incidents were actually removed by the spatial analysis technique. In particular, the first four rows from Table 4, which contained claims about the increase in traffic of Google, were discarded since the keywords Internet and Google were spatially present across all the 3 cities on this particular day. Table 7 shows the top 10 tweets with high information gain along with credibility and spatial analysis. The inclusion of credibility part with the spatial analysis improves the results by moving up credible incident related events as can be observed by comparing the Tables 6 and 7 respectively. In Table 7 the tweets generated by random users were pushed down in the list and those supplying more information moved to top as they happen to be associated with a higher truth value. 
Table 7: Top 10 tweets from San Diego with high Information Gain, Credibility and Spatial Analysis

\begin{tabular}{|c|c|c|}
\hline Rank & Tweets & Keyword Pair \\
\hline 1 & $\begin{array}{l}\text { TRAFFIC ALERT: At least } 1 \text { person hurt after crash with Sycuan bus. } \\
\text { Dehesa Road is closed. http://t.co/iXdRCfvFlG http://t.co/9FhmKl2BGd }\end{array}$ & (alert, dehesa) \\
\hline 2 & $\begin{array}{l}\text { TRAFFIC: A passenger bus collided with a car on Dehesa Rd. near } \\
\text { \#Sycuan casino: http://t.co/MqGIvS0vsU http://t.co/2cytXqMiET }\end{array}$ & (casino, dehesa) \\
\hline 3 & $\begin{array}{l}\text { Traffic Alert: Casino bus crashes with car - Dehesa Rd. closed } \\
\text { http://t.co/eQ9UCMF8D8 }\end{array}$ & (alert, dehesa) \\
\hline 4 & $\begin{array}{l}\text { Culture Report: Foot Traffic Way Up at One Balboa Park Museum } \\
\text { http://t.co/Fn9d9nQG6r }\end{array}$ & (balboa, museum) \\
\hline 5 & $\begin{array}{l}\text { Think I'll stop by the \#Timken too! RT @ voiceofsandiego Culture Report: } \\
\text { Foot Traffic Way Up at One Balboa Park Museum http://t.co/oucClyqeKJ }\end{array}$ & (balboa, museum) \\
\hline 6 & $\begin{array}{l}\text { Lights out on part of \#BayBridge! @ abc7newsBayArea reporting } \\
\text { \#CalTrans is aware. No traffic impact. http://t.co//zoM6QsI2x }\end{array}$ & (baybridge, caltrans) \\
\hline 7 & $\begin{array}{l}\text { Thank The Lord traffic is a million times better than yesterday! I got to } \\
\text { work yesterday with } 30 \text { seconds to spare lol }\end{array}$ & (million, seconds) \\
\hline 8 & i swear, twitters like a steele canyon traffic tracker.. & (tracker, swear) \\
\hline 9 & $\begin{array}{l}\text { Man Named Beezow Doo-doo Zopittybop-bop-bop Arrested: Man with } \\
\text { unusual name charged after a recent traffic sto... http://t.co/NO7EaxwR0P }\end{array}$ & (zopittybop, beezow) \\
\hline 10 & $\begin{array}{l}\text { Can't wait till people start dropping their classes. I'm not sure how much } \\
\text { longer I can deal with this traffic. Mesa, you suck. }\end{array}$ & (start, people) \\
\hline
\end{tabular}

\subsubsection{Matching with Sensor Anomalies}

It remains to match the anomalous feeds found using the above technique with the sensor anomalies. To explain a particular sensor anomaly, we consider only those feeds that occurred within the 24 hours following the anomaly. We then search for a location keyword match between the feeds and the sensor anomalies. To match locations, we consider the geo-keywords associated with each physical sensor. These keywords refer to the local landmarks that people would typically use when describing the location in question. An automated way of generating these would be to use Google's "what's here" interface at each sensor location. For each anomalous sensor cluster, we combine the location keywords associated with each of that cluster's sensors. These keywords become the location indicators of the corresponding anomalous event. In the case of traffic anomalies, location keywords associated with a sensor usually include the highway number (of the highway that the sensor monitors), exit names (near the sensor), as well as names of nearby important landmarks. While we hand-picked the geo-keywords associated with each sensor in this paper, a machine learning approach has been described in recent literature [6] to automate finding keywords that refer to specific locations. This approach could be used to automate the process for larger systems. Based on the matches found using the landmarks and other keywords, all the explanations are provided as map pin-points to the user as the final output.

Discriminative feeds that occur within 24 hours from a given event and that match its location (i.e., contain one or more of its location keywords) are sorted by information gain with credibility scores. We then simply choose the top feeds as the likely explanations of the sensor anomaly. In the evaluation section, we show that on average less than two microblog entries are needed from the top of the sorted list to find the correct explanation. The complete algorithm to explain sensor anomalies using social feeds is described below.

Algorithm 1 analyzes feeds from current window and previous window to find the pair of words in the current window which have a high information gain contributing 


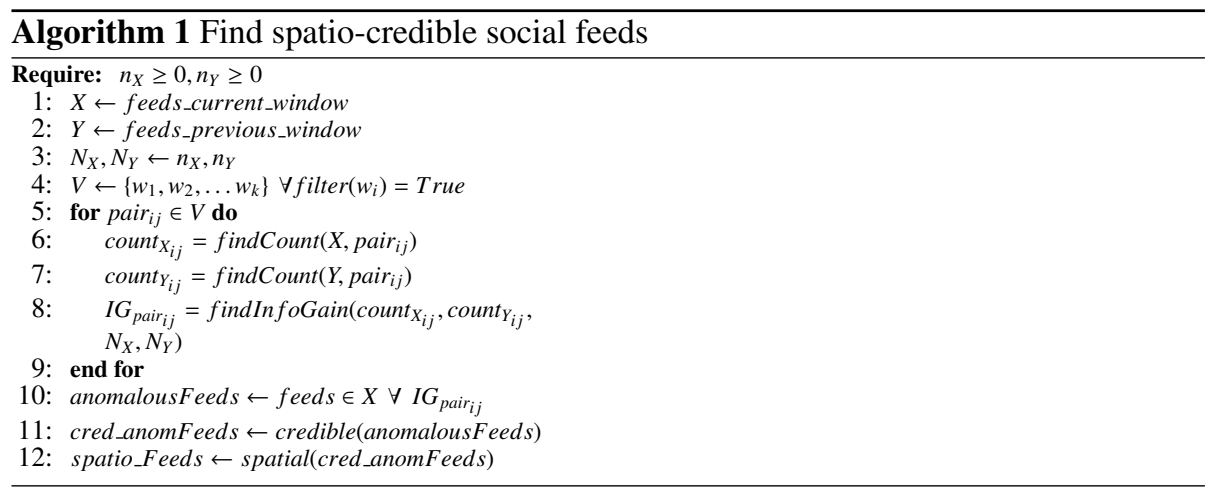

to anomalous feeds which are then filtered based on credibility and spatial analysis to give the final set of candidate explanations. The variables $X$ and $Y$ represent feeds in respective windows with length $N_{X}$ and $N_{Y} . V$ is the vocabulary of single keywords. The filtering function filter removes all punctuation marks and returns only words with a minimum length of 4 letters that have been tagged as Nouns by the Part-of-Speech tagger. The findCount function finds the count of a pair $_{i j}$ in a given window. Using the counts from each window the information gain can be calculated using the function findInfoGain using Equation (2). These anomalous items are ranked using a function credible using Equation (6). At last these credible anomalous items are analysed by a function spatial which removes all those that do not contain spatially localised keywords and return the final set of candidates which can be possible explanations for sensor anomalies.

\section{Implementation and Evaluation}

In the traffic data sets, the various types of incidents included accidents, breakdowns, congestion, hazards, police activity, weather and other types. Table 8 shows the percentage of incidents of different durations observed during the 3-week testing period for each city. To reduce the number of events considered, we restrict our evaluation to events of at least a 100-minute duration. This restriction was made due to two reasons - (i) the Twitter search API provides limited access to the data $(\sim 1 \%)$ from the main servers, so we focus on major events involving larger user participation on the social side; (ii) to make the evaluation manageable as manual labor was involved to compare the automated explanations with the ground truth for each event. The ground truth data was available from PEMS database system for every sensor on a particular given date. Fig 3 shows the distribution of unique tweets for each city on each date during this 3-week period.

The evaluation consists of two parts. First, we measure recall by considering how many of the observed incidents were explained by our algorithm. Second, we measure precision by computing the average rank of the correct explanation on the returned ranked list of possible explanations for each incident. 
Table 8: Duration and percentage of incidents

\begin{tabular}{|c|c|c|c|c|c|c|c|c|c|c|c|c|c|c|}
\hline City/Minutes & $<60$ & 60 & 65 & 70 & 75 & 80 & 85 & 90 & 95 & 100 & 105 & 110 & 115 & $\geq 120$ \\
\hline Los Angeles & $86 \%$ & $2 \%$ & $2.2 \%$ & $1.1 \%$ & $1.2 \%$ & $1.2 \%$ & $0.6 \%$ & $0.5 \%$ & $0.7 \%$ & $0.3 \%$ & $0.4 \%$ & $0.3 \%$ & $0.3 \%$ & $3.2 \%$ \\
\hline San Diego & $87.3 \%$ & $1.9 \%$ & $1.4 \%$ & $1.1 \%$ & $0.9 \%$ & $0.7 \%$ & $0.6 \%$ & $0.4 \%$ & $0.8 \%$ & $0.2 \%$ & $0.4 \%$ & $0.4 \%$ & $0.4 \%$ & $3.5 \%$ \\
\hline San Francisco & $87.9 \%$ & $0.9 \%$ & $0.5 \%$ & $1.6 \%$ & $0.7 \%$ & $1.6 \%$ & $0.5 \%$ & $0.7 \%$ & $0.2 \%$ & $0.5 \%$ & $0 \%$ & $0.2 \%$ & $0.5 \%$ & $4.2 \%$ \\
\hline
\end{tabular}

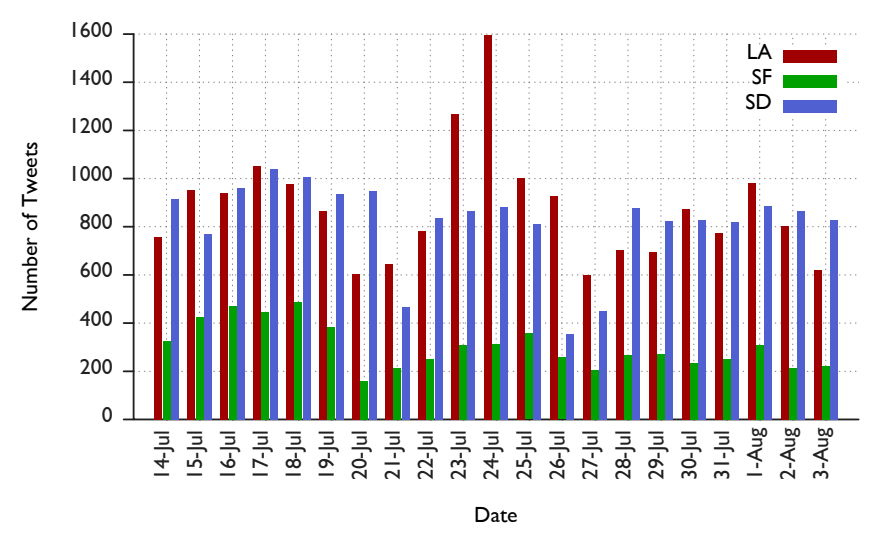

Figure 3: Distribution of tweets

\subsection{Evaluation of Recall}

In order to evaluate the recall, we determine the number of events that were correctly explained among all the feeds that were returned by our enhanced algorithm on each day of the 3-week period for each city. The correct explanations were determined on the basis of timestamp of the feeds and the keywords used to describe local landmarks or freeway numbers around the physical sensors. These events included categories such as accidents, breakdown, congestion, and other. Fig 3 shows the distribution of unique tweets collected each day (i.e., ignoring retweets) in each of the three cities that contained the keyword "traffic". Note that, our tweet collection software used the Twitter search API, which is subject to rate-limiting by Twitter. Hence, the collected tweets do not represent the entire set of tweets actually generated on the social network. Nevertheless, it is sufficient for purposes of testing our algorithm. Note that San Francisco, on average, had relatively fewer tweets compared to Los Angeles and San Diego, whereas Los Angeles had the most.

Since tweets are the source that our algorithm uses for explaining sensor anomalies, clearly no matter how good or bad the anomaly detection and explanation algorithm is, it will not explain anomalies that no one tweeted about. Hence, of the anomalies recorded by sensors, we first manually tallied those that had a Twitter presence (i.e., those that someone tweeted about), then determined how many of these were correctly explained by our algorithm.

Table 9, 10 and 11 show, in the first column, labeled "Total", the total number of unique tweets collected with keyword "Traffic", for each day for each city. It shows in the second column, labeled "Relevant", the number of tweets that were related to traffic incidents. These relevant tweets actually contained descriptions of traffic events. 
Table 9: San Diego: Incident Related tweets \& Identified Tweets

\begin{tabular}{|c|c|c|c|c|c|c|c|c|c|c|c|c|c|c|c|c|c|c|c|c|}
\hline \multirow[b]{2}{*}{ Date } & \multirow[b]{2}{*}{ Total } & \multirow[b]{2}{*}{ Relevant } & \multicolumn{3}{|c|}{ Accident } & \multicolumn{3}{|c|}{ Hazard } & \multicolumn{3}{|c|}{ Breakdown } & \multicolumn{3}{|c|}{ Weather } & \multicolumn{3}{|c|}{ Other } & \multicolumn{3}{|c|}{ Total } \\
\hline & & & $\mathrm{O}$ & $\mathrm{T}$ & ID & $\mathrm{O}$ & $\mathrm{T}$ & ID & $\mathrm{O}$ & $\mathrm{T}$ & ID & $\mathrm{O}$ & $\mathrm{T}$ & ID & $\mathrm{O}$ & $\mathrm{T}$ & ID & $\mathrm{O}$ & $\mathrm{T}$ & ID \\
\hline $7 / 14$ & 914 & 61 & 1 & 0 & 0 & 1 & 0 & 0 & 0 & 0 & 0 & 0 & 0 & 0 & 1 & 0 & 0 & 3 & 0 & 0 \\
\hline $7 / 15$ & 770 & 45 & 0 & 0 & 0 & 1 & 0 & 0 & 0 & 0 & 0 & 0 & 0 & 0 & 1 & 0 & 0 & 2 & 0 & 0 \\
\hline $7 / 16$ & 961 & 97 & 3 & 2 & 1 & 3 & 2 & 2 & 0 & 0 & 0 & 0 & 0 & 0 & 0 & 0 & 0 & 6 & 4 & 3 \\
\hline $7 / 17$ & 1038 & 117 & 4 & 1 & 1 & 0 & 0 & 0 & 0 & 0 & 0 & 0 & 0 & 0 & 2 & 0 & 0 & 6 & 1 & 1 \\
\hline $7 / 18$ & 1007 & 114 & 2 & 1 & 1 & 1 & 0 & 0 & 0 & 0 & 0 & 0 & 0 & 0 & 0 & 0 & 0 & 3 & 1 & 1 \\
\hline $7 / 19$ & 937 & 83 & 2 & 0 & 0 & 2 & 2 & 2 & 0 & 0 & 0 & 0 & 0 & 0 & 0 & 0 & 0 & 4 & 2 & 2 \\
\hline $7 / 20$ & 949 & 37 & 2 & 0 & 0 & 1 & 0 & 0 & 0 & 0 & 0 & 0 & 0 & 0 & 0 & 0 & 0 & 3 & 0 & 0 \\
\hline $7 / 22$ & 837 & 66 & 1 & 1 & 1 & 1 & 0 & 0 & 0 & 0 & 0 & 0 & 0 & 0 & 0 & 0 & 0 & 2 & 1 & 1 \\
\hline $7 / 23$ & 865 & 102 & 2 & 0 & 0 & 2 & 0 & 0 & 0 & 0 & 0 & 0 & 0 & 0 & 3 & 2 & 1 & 7 & 2 & 1 \\
\hline $7 / 24$ & 881 & 108 & 2 & 0 & 0 & 1 & 0 & 0 & 0 & 0 & 0 & 0 & 0 & 0 & 1 & 0 & 0 & 4 & 0 & 0 \\
\hline $7 / 25$ & 812 & 83 & 5 & 2 & 2 & 2 & 0 & 0 & 0 & 0 & 0 & 0 & 0 & 0 & 0 & 0 & 0 & 7 & 2 & 2 \\
\hline $7 / 26$ & 353 & 50 & 1 & 1 & 1 & 1 & 0 & 0 & 1 & 0 & 0 & 0 & 0 & 0 & 0 & 0 & 0 & 3 & 1 & 1 \\
\hline $7 / 27$ & 451 & 64 & 1 & 0 & 0 & 2 & 1 & 1 & 0 & 0 & 0 & 0 & 0 & 0 & 3 & 1 & 1 & 6 & 2 & 2 \\
\hline $7 / 28$ & 876 & 68 & 0 & 0 & 0 & 1 & 0 & 0 & 0 & 0 & 0 & 0 & 0 & 0 & 1 & 0 & 0 & 2 & 0 & 0 \\
\hline $7 / 29$ & 823 & 77 & 1 & 0 & 0 & 1 & 0 & 0 & 0 & 0 & 0 & 0 & 0 & 0 & 1 & 0 & 0 & 3 & 0 & 0 \\
\hline $7 / 30$ & 826 & 54 & 3 & 1 & 1 & 2 & 1 & 1 & 0 & 0 & 0 & 0 & 0 & 0 & 0 & 0 & 0 & 5 & 2 & 2 \\
\hline $8 / 1$ & 886 & 68 & 1 & 1 & 1 & 1 & 1 & 1 & 0 & 0 & 0 & 0 & 0 & 0 & 0 & 0 & 0 & 2 & 2 & 2 \\
\hline $8 / 2$ & 866 & 48 & 3 & 2 & 2 & 1 & 0 & 0 & 0 & 0 & 0 & 0 & 0 & 0 & 1 & 1 & 1 & 5 & 3 & 3 \\
\hline $8 / 3$ & 829 & 16 & 3 & 1 & 0 & 0 & 0 & 0 & 0 & 0 & 0 & 1 & 0 & 0 & 0 & 0 & 0 & 4 & 1 & 0 \\
\hline Total & 15881 & 1358 & 37 & 13 & 11 & 24 & 7 & 7 & 1 & 0 & 0 & 1 & 0 & 0 & 14 & 4 & 3 & 77 & 24 & 21 \\
\hline
\end{tabular}

Table 10: San Francisco: Incident Related tweets \& Identified Tweets

\begin{tabular}{|c|c|c|c|c|c|c|c|c|c|c|c|c|c|c|c|c|c|c|c|c|}
\hline \multirow[b]{2}{*}{ Date } & \multirow[b]{2}{*}{ Total } & \multirow[b]{2}{*}{ Relevant } & \multicolumn{3}{|c|}{ Accident } & \multicolumn{3}{|c|}{ Hazard } & \multicolumn{3}{|c|}{ Breakdown } & \multicolumn{3}{|c|}{ Weather } & \multicolumn{3}{|c|}{ Other } & \multicolumn{3}{|c|}{ Total } \\
\hline & & & $\mathrm{O}$ & $\mathrm{T}$ & ID & $\mathrm{O}$ & $\mathrm{T}$ & ID & $\mathrm{O}$ & $\mathrm{T}$ & ID & $\mathrm{O}$ & $\mathrm{T}$ & ID & $\mathrm{O}$ & $\mathrm{T}$ & ID & $\mathrm{O}$ & $\mathrm{T}$ & ID \\
\hline $7 / 17$ & 445 & 50 & 1 & 1 & 1 & 0 & 0 & 0 & 0 & 0 & 0 & 0 & 0 & 0 & 0 & 0 & 0 & 1 & 1 & 1 \\
\hline $7 / 18$ & 489 & 24 & 0 & 0 & 0 & 1 & 1 & 1 & 0 & 0 & 0 & 0 & 0 & 0 & 0 & 0 & 0 & 1 & 1 & 1 \\
\hline $7 / 19$ & 383 & 22 & 0 & 0 & 0 & 0 & 0 & 0 & 0 & 0 & 0 & 0 & 0 & 0 & 0 & 0 & 0 & 0 & 0 & 0 \\
\hline $7 / 20$ & 158 & 12 & 0 & 0 & 0 & 0 & 0 & 0 & 0 & 0 & 0 & 1 & 0 & 0 & 0 & 0 & 0 & 1 & 0 & 0 \\
\hline $7 / 21$ & 214 & 22 & 0 & 0 & 0 & 3 & 0 & 0 & 0 & 0 & 0 & 0 & 0 & 0 & 0 & 0 & 0 & 3 & 0 & 0 \\
\hline $7 / 22$ & 251 & 31 & 2 & 0 & 0 & 2 & 2 & 2 & 0 & 0 & 0 & 0 & 0 & 0 & 0 & 0 & 0 & 4 & 2 & 2 \\
\hline $7 / 26$ & 257 & 41 & 0 & 0 & 0 & 1 & 1 & 1 & 0 & 0 & 0 & 0 & 0 & 0 & 0 & 0 & 0 & 1 & 1 & 1 \\
\hline $7 / 28$ & 269 & 42 & 2 & 0 & 0 & 1 & 1 & 1 & 0 & 0 & 0 & 0 & 0 & 0 & 0 & 0 & 0 & 3 & 1 & 1 \\
\hline $7 / 29$ & 270 & 32 & 0 & 0 & 0 & 1 & 0 & 0 & 0 & 0 & 0 & 0 & 0 & 0 & 1 & 1 & 0 & 2 & 1 & 0 \\
\hline $7 / 30$ & 235 & 16 & 0 & 0 & 0 & 1 & 1 & 1 & 0 & 0 & 0 & 0 & 0 & 0 & 0 & 0 & 0 & 1 & 1 & 1 \\
\hline $7 / 31$ & 252 & 32 & 1 & 0 & 0 & 0 & 0 & 0 & 0 & 0 & 0 & 0 & 0 & 0 & 1 & 1 & 1 & 2 & 1 & 1 \\
\hline $8 / 1$ & 310 & 53 & 1 & 1 & 0 & 0 & 0 & 0 & 0 & 0 & 0 & 0 & 0 & 0 & 0 & 0 & 0 & 1 & 1 & 0 \\
\hline $8 / 2$ & 212 & 35 & 2 & 0 & 0 & 0 & 0 & 0 & 0 & 0 & 0 & 0 & 0 & 0 & 0 & 0 & 0 & 2 & 0 & 0 \\
\hline Total & 3745 & 412 & 9 & 2 & 1 & 10 & 6 & 6 & 0 & 0 & 0 & 1 & 0 & 0 & 2 & 2 & 1 & 22 & 10 & 8 \\
\hline
\end{tabular}

For each type of incident, there are three columns; the first, denoted $O$, represents the number of incidents that were observed in the physical sensor network; the second, denoted $T$, represents the number of incidents that were tweeted about; and the third, called $I D$ represents the number of events that were automatically explained by our algorithm. Since it is impossible for our algorithm to explain something that is not tweeted about, we focus on the ratio $I D / T$, which we term recall. Note that some dates are missing from these tables. The missing dates are those where no anomalies were observed by sensors.

From the table, it can be seen that a $100 \%$ recall is achieved for Hazard-related incidents and $84 \%$ for Accident-related incidents in San Diego. Also, 100\% recall is achieved for Hazard-related incidents (and only one Accident-related event was missed) in San Francisco. Finally, 83\% recall was achieved for Hazard-related incidents and $73 \%$ for Accident-related events in Los Angeles. These high recall values 
Table 11: Los Angeles: Incident Related tweets \& Identified Tweets

\begin{tabular}{|c|c|c|c|c|c|c|c|c|c|c|c|c|c|c|c|c|c|c|c|c|}
\hline \multirow[b]{2}{*}{ Date } & \multirow[b]{2}{*}{ Total } & \multirow[b]{2}{*}{ Relevant } & \multicolumn{3}{|c|}{ Accident } & \multicolumn{3}{|c|}{ Hazard } & \multicolumn{3}{|c|}{ Breakdown } & \multicolumn{3}{|c|}{ Weather } & \multicolumn{3}{|c|}{ Other } & \multicolumn{3}{|c|}{ Total } \\
\hline & & & $\mathrm{O}$ & $\mathrm{T}$ & ID & $\mathrm{O}$ & $\mathrm{T}$ & ID & $\mathrm{O}$ & $\mathrm{T}$ & ID & $\mathrm{O}$ & $\mathrm{T}$ & ID & $\mathrm{O}$ & $\mathrm{T}$ & ID & $\mathrm{O}$ & $\mathrm{T}$ & ID \\
\hline $7 / 14$ & 759 & 95 & 5 & 3 & 2 & 2 & 1 & 1 & 0 & 0 & 0 & 0 & 0 & 0 & 0 & 0 & 0 & 7 & 4 & 3 \\
\hline $7 / 15$ & 952 & 151 & 4 & 1 & 0 & 6 & 4 & 4 & 0 & 0 & 0 & 0 & 0 & 0 & 0 & 0 & 0 & 10 & 5 & 4 \\
\hline $7 / 16$ & 941 & 101 & 2 & 1 & 0 & 4 & 2 & 2 & 0 & 0 & 0 & 0 & 0 & 0 & 2 & 1 & 0 & 8 & 4 & 2 \\
\hline $7 / 17$ & 1050 & 72 & 4 & 1 & 1 & 4 & 2 & 1 & 0 & 0 & 0 & 0 & 0 & 0 & 0 & 0 & 0 & 8 & 3 & 2 \\
\hline $7 / 18$ & 979 & 102 & 4 & 1 & 0 & 2 & 1 & 1 & 0 & 0 & 0 & 0 & 0 & 0 & 2 & 0 & 0 & 8 & 2 & 1 \\
\hline $7 / 19$ & 864 & 48 & 0 & 0 & 0 & 2 & 1 & 1 & 0 & 0 & 0 & 0 & 0 & 0 & 3 & 0 & 0 & 5 & 1 & 1 \\
\hline $7 / 20$ & 604 & 48 & 4 & 0 & 0 & 1 & 0 & 0 & 0 & 0 & 0 & 0 & 0 & 0 & 0 & 0 & 0 & 5 & 0 & 0 \\
\hline $7 / 21$ & 644 & 108 & 6 & 2 & 2 & 1 & 1 & 1 & 0 & 0 & 0 & 0 & 0 & 0 & 0 & 0 & 0 & 7 & 3 & 3 \\
\hline $7 / 22$ & 782 & 102 & 3 & 1 & 1 & 3 & 1 & 1 & 0 & 0 & 0 & 0 & 0 & 0 & 1 & 0 & 0 & 7 & 2 & 2 \\
\hline $7 / 23$ & 1266 & 286 & 2 & 0 & 0 & 3 & 2 & 0 & 0 & 0 & 0 & 0 & 0 & 0 & 1 & 1 & 1 & 6 & 3 & 1 \\
\hline $7 / 24$ & 1596 & 91 & 1 & 0 & 0 & 1 & 0 & 0 & 2 & 1 & 1 & 0 & 0 & 0 & 1 & 1 & 0 & 5 & 2 & 1 \\
\hline $7 / 25$ & 1002 & 141 & 1 & 0 & 0 & 2 & 2 & 2 & 0 & 0 & 0 & 0 & 0 & 0 & 0 & 0 & 0 & 3 & 2 & 2 \\
\hline $7 / 26$ & 928 & 71 & 6 & 3 & 3 & 1 & 0 & 0 & 0 & 0 & 0 & 0 & 0 & 0 & 2 & 0 & 0 & 9 & 3 & 3 \\
\hline $7 / 27$ & 599 & 63 & 8 & 1 & 1 & 2 & 0 & 0 & 0 & 0 & 0 & 0 & 0 & 0 & 0 & 0 & 0 & 10 & 1 & 1 \\
\hline $7 / 28$ & 705 & 55 & 1 & 0 & 0 & 3 & 1 & 1 & 0 & 0 & 0 & 0 & 0 & 0 & 0 & 0 & 0 & 4 & 1 & 1 \\
\hline $7 / 29$ & 694 & 140 & 3 & 1 & 1 & 3 & 1 & 0 & 0 & 0 & 0 & 0 & 0 & 0 & 0 & 0 & 0 & 6 & 2 & 1 \\
\hline $7 / 30$ & 875 & 99 & 6 & 2 & 2 & 1 & 1 & 1 & 0 & 0 & 0 & 0 & 0 & 0 & 0 & 0 & 0 & 7 & 3 & 3 \\
\hline $7 / 31$ & 775 & 108 & 4 & 2 & 1 & 4 & 3 & 3 & 1 & 0 & 0 & 0 & 0 & 0 & 0 & 0 & 0 & 9 & 5 & 4 \\
\hline $8 / 1$ & 982 & 144 & 3 & 1 & 0 & 1 & 1 & 1 & 0 & 0 & 0 & 0 & 0 & 0 & 1 & 1 & 1 & 5 & 3 & 2 \\
\hline $8 / 2$ & 803 & 78 & 4 & 1 & 1 & 2 & 0 & 0 & 0 & 0 & 0 & 0 & 0 & 0 & 2 & 0 & 0 & 8 & 1 & 1 \\
\hline $8 / 3$ & 619 & 89 & 4 & 1 & 1 & 0 & 0 & 0 & 0 & 0 & 0 & 0 & 0 & 0 & 0 & 0 & 0 & 4 & 1 & 1 \\
\hline Total & 18419 & 2192 & 75 & 22 & 16 & 48 & 24 & 20 & 3 & 1 & 1 & 0 & 0 & 0 & 15 & 4 & 2 & 141 & 51 & 39 \\
\hline
\end{tabular}

indicate that our information gain algorithm performs well in explaining events if the explanation is actually present on Twitter. We expect many more events to have Twitter presence than what we were able to collect, but we did not have access to the full Twitter Firehose . Morover, as the popularity of social networks grows, more information will be available, and recall will further increase.

\subsection{Evaluation of Precision}

To evaluate precision of our algorithm, we determine how good it is at picking the right explanation for the anomalies at hand. Since the algorithm returns a ranked list of possible explanations for each physical anomaly, to evaluate precision, we simply compute how far down the list the correct explanation is. We call it the mean rank (of correct explanations).

We compare the mean rank when tweets (i.e., candidate explanations) are sorted by four different algorithms: (i) the information gain, which corresponds to our initial method described in [7], (ii) information gain with credibility extensions, (iii) information gain with spatial extensions, and (iv) information gain with both credibility and spatial extensions. For each sensor anomaly, we find two ranks. First, we find the rank of the explanation of that anomaly on the list containing all explanations of all anomalies. The average such rank across all anomalies is called Avg Rank. We also determine, for each sensor anomaly, the rank of the correct explanation of the anomaly on a list restricted to tweets with keywords referring to the location of the corresponding sensor. The average such rank across all anomalies is called Sp Rank.

Table 12 shows the average rank obtained for each city using the different algorithms. It can be seen from the table that the Avg Rank values generally improve as we consider credibility and location. San Diego is an exception, where the credibility 
Table 12: Precision

\begin{tabular}{|c|c|c|c|c|c|c|c|c|}
\hline \multirow{2}{*}{ City } & \multicolumn{2}{|c|}{ IG } & \multicolumn{2}{c|}{ Spatio-IG } & \multicolumn{2}{c|}{ Cred-IG } & \multicolumn{2}{c|}{ Spatio-Cred-IG } \\
\cline { 2 - 9 }$y$ & Avg Rank & Sp Rank & Avg Rank & Sp Rank & Avg Rank & Sp Rank & Avg Rank & Sp Rank \\
\hline Los Angeles & 23.071 & 1.4285 & 20.785 & 1.357 & 17.785 & 1.357 & 15.642 & 1.285 \\
\hline San Francisco & 20.4 & 1.6 & 19.2 & 1.6 & 5.4 & 1.2 & 5.2 & 1 \\
\hline San Diego & 13.785 & 1.142 & 12.285 & 1.071 & 11.857 & 1.214 & 11.071 & 1.214 \\
\hline
\end{tabular}

Table 13: Percentage of times for Top Tweet

\begin{tabular}{|c|c|c|c|c|}
\hline City & IG & Spatio-IG & Cred-IG & Spatio-Cred-IG \\
\hline Los Angeles & $71.7 \%$ & $76.9 \%$ & $76.9 \%$ & $89.7 \%$ \\
\hline San Francisco & $75 \%$ & $75 \%$ & $87.5 \%$ & $100 \%$ \\
\hline San Diego & $85.71 \%$ & $95.2 \%$ & $76.19 \%$ & $76.19 \%$ \\
\hline
\end{tabular}

extension was not useful. This is attributed to the small size of the data set making it harder to estimated credibility correctly using statistical means. The spatial extension alone was found more useful in this case. Note also that the Sp Rank is close to 1 in most cases (and is always below 2), which means that the correct explanation was is found on average among the top two tweets, when the tweets are ranked by information gain (or its extensions) and matched to locations using location keywords.

The above evaluation shows that the approach has promise at explaining major anomalies by using a very simple yet robust technique. The correct explanation was usually one of the top two candidates returned. Finally, as social network use becomes more pervasive, the approach is expected to become more effective.

\section{State of the Art}

A significant body of literature exists on anomaly detection [8] and diagnostics. Much of the work on anomaly detection focuses on identifying low-probability patterns. In physical sensing systems, those patterns may include unusual combinations of sensor readings. In social networks, those patterns may include unusual topological features or dynamics. Anomaly detection has also been used extensively in security literature. For example, it was used for intrusion detection [9] or to identify different types of attackes, such as Sybil attacks [10]. Literature on failure diagnostics used anomaly detection techniques in the context of debugging, where the occurrence of unusual behavior of the tested program or system is correlated with unusual execution paths to identify paths that might be "buggy" causing the behavior problem to occur [11]. The above extensive and multidisciplinary body of literature is not the objective in this paper. Instead our objective is to automatically find microblog entries that best explain sensor anomalies. Hence, we focus on finding text entries that may explain local events. The work must detect entries that are (i) discriminative, (ii) credible, and (iii) spatially relevant. Ideally, it should not rely on understanding text semantics in order to be easily applicable regardless of language.

The applications served by this paper fall in the domain of social sensing, where the objective is to collect data from human sources (or their sensors) that maps some physical variable, phenomenon, or event, except that in our case, we focus on collecting explanations of unusual events. For instance, the Nericell project [12] presents a 
system that performs rich sensing using smartphones to monitor road and traffic conditions. The GreenGPS system [13] provides a service that computes fuel-efficient routes for vehicles by exploiting current traffic conditions. Separately, much research focused on detecting events from social media. A real-time event detection framework was recently described [14] using Twitter as the social network. The work presents the challenges incurred primarily due to high data volume. Another effort [15], related to event identification in Twitter, uses a classification-based approach to generate a learning model used for classifying an incoming stream of data as event and nonevent messages. A system that tracks mentions of specific health conditions was also reported [16], including over a dozen ailments, allergies, obesity issues, and insomnia. In work most similar to ours [17], a novel event retrieval framework is presented, where both the contents of tweets and the volume of the microblogging activity are exploited to locate an event that matches a user's interests. The framework measures unusual microblogging activities in an area and uses that as an indication of the occurrence of an event. Although most of these efforts are related to detecting events either in the sensor network or the social network domain, none of them focuses on the joint usage of the two networks to corroborate the detection of event on one network (primarily sensor networks) with those present in another network. We have made a successful effort towards this approach and demonstrated the feasibility with the implementation and evaluation of such a system.

Previous work [18] addressed identifying specific anomalous events by tracing trajectories reported by humans and finding the cause using social media. In ClariSense [7] we used a different approach for identifying discriminative social feeds and, at the same time, enable the service to support various types of events. We built a generic system which aimed at jointly exploiting physical sensors and social feeds to both detect and explain anomalies using the information gain algorithm, which relied on frequency of keyword pairs to find discriminative feeds in a window set. As an extension, we have made an effort towards improving the efficiency of our system to get better recall and precision values. The major components of our present contribution is the addition of a credibility score with the information gain algorithm followed by a spatial analysis technique to remove feeds out of the local scope of interest. To further support our contribution, we have included evaluations for all types of anomalies observed in the road traffic network without any restriction over particular incident types. Thus, our system demonstrates a feasible solution to the objective of our research work in a simple yet robust way.

\section{Discussion and Lessons Learned}

In this section we present lessons learned from traffic data collected from sensor and social networks. We also briefy discuss the advantages and limitations of such a service. Our first observation lies in the importance of clustering of nearby sensors that exhibit anomalous reports. Clustering is important in order to identify the correct number of events that need to be explained. Often anomalous readings of multiple sensors are attributed to the same event. For example a major traffic incident may result in blockage of a segment of a freeway, causing anomalous traffic patterns to be reported by multiple sensors on the way. All such sensors should be clustered to denote 
a single event that needs a single explanation. Otherwise, false positives and redundant events may be generated.

Second, while location is a key feature in matching social microblogs with sensors reporting anomalous readings, we found that relying on tweet (location) geotags is not productive. First, only a few percent of tweets are geotaagged. Second, often people tweet later when they are far from the location they tweet about. Hence location of the reporting source is a poor indicator of location of the reported event. Instead, techniques are needed that extract locations and landmarks referred to in the text of the tweets. For example, freeways, street intersections, and exit numbers are often used by the sources when referring to an event.

Third, data showed that most references to the same traffic accident contained the same set of 2-3 keyowrds associated with the event (which we defined as event signature), creating a set of keyword combinations that occured disproportionately around the time of the event and characterized tweets about the event. This property simplified the task of identifying new events (simply by finding new keyword pairs of high information gain), without requiring that keywords characteristic to each event be known in advance. Once high-information-gain keywords were identified, one simply associated tweets with "bins" based on keyword pairs they contained. Tweets about different events would thus fall into different bins. In general, the amount of data related to an event was proportional to the severity of event.

We should reiterate, at this point, that while the paper described and evaluated a service for explaining anomalous traffic conditions, we do not necessaily claim that this service is the ultimate contribution of the paper, nor do we claim that it is better than obvious alternatives such as listening to news. In fact, quite the opposite; we chose this particular case study because ground truth is readily available by other means, making a systematic evaluation possible. We hope, however, that the ideas in this paper are not exclusively applicable to the traffic domain. While algorithms such as clustering of anomalous reports may use different thresholds that need to be trained for the target domain, the key insights that (i) information gain can be used to identify keyword combinations specific to new events, and that (ii) such combinations can in turn help find tweets about the events, seem to transcend the traffic domain. Hence, the intended contribution is to facilitate follow-up work that establishes the applicability of the approach in other settings, where ground truth may be harder to come by, making reliance on social networks more meaningful.

Our service requires very little manual customization and bootstrapping. All that is needed for the system to work is a suitably filtered Twitter feed that offers the input tweets for our algorithm to look at. In the Traffic case study presented in this paper, tweets were filtered using the keyword "Traffic" (i.e., only tweets containing that word were retained). In other contexts, such as, for example, monitoring and explaining pollution or radiation measurements, one might use the keyword "Pollution" or "Radiation", respectively, to filter the Twitter stream. Another bootstrapping issue relates to matching sensors to location references in tweets in order to identify tweets that refer to the particular sensor location. It requires associating each sensor with a set of words that a tweet might contain when referring to the corresponding location. For example, a traffic sensor near a stadium should be associated with the landmark name "Stadium", as well as the name of the stadium and the name of the street or exit that the sensor is lo- 
cated at. This association can be done automatically using a reverse geocoding service (such as the one offered by Google). The service translates GPS coordinates into a text address and allows one to obtain the list of place IDs associated with the given coordinates. Place IDs include nearby landmarks, freeways, exits, and businesses. Hence, the list of location keywords for each sensor can be populated without manual intervention, allowing the service to scale to large numbers of sensors. Our prior work also addressed the challenge of translating text references in tweets to location coordinates, which can also be used in matching location of sensors to tweets [19].

Through this work we established that our system is capable of identifying causes of physical anomalies in vehicular traffic flow using social networks. The wide popularity of social networks, where content is posted in real time makes it possible to detect some events even before they are picked up by news agencies. This is especially true of events that occur in busy urban spaces, where they can be observed by a large number of users. Protests, demonstration, flash crowds, and physical damage events (e.g., residential fires, shootings, violence, or street flooding) fall into that category. The approach is less applicable to detection of rural events, or events in unpopulated spaces. Hence, although the explanation and experiments with the system in this paper were based on traffic data, we expect the work to be applicable to other urban domains.

\section{Conclusions}

In this paper, we demonstrated the feasibility of explaining likely causes of traffic anomalies by identifying unusual social network feeds that are correlated with each anomaly in time and in space. An in-depth study to evaluate our system was performed on vehicular traffic datasets in three big cities from California. Our system performed well in this scenario, especially for Hazard- and Accident-related incidents. The mean rank of the correct explanations was less than two, meaning that the right explanation was contained in the top two selected microblog entries on average. The researchers are currently working on extensions that test scalable anomaly detection and explanation in real time beyond the domain of traffic incidents. Finally, it is interesting to investigate whether it may be possible to predict physical anomalies using social network feeds. These extensions are delegated to future work.

\section{Acknowledgements}

Research reported in this paper was sponsored by the Army Research Laboratory and was accomplished under Cooperative Agreement W911NF-09-2-0053, DTRA grant HDTRA1-10-1- 0120, and NSF grants NSF CNS 13-29886, CNS 09-58314, and CNS 10-35736. The views and conclusions contained in this document are those of the authors and should not be interpreted as representing the official policies, either expressed or implied, of the Army Research Laboratory or the U.S. Government. The U.S. Government is authorized to reproduce and distribute reprints for Government purposes notwithstanding any copyright notation here on. 


\section{References}

[1] Twitter,http://www.twitter.com/.

[2] Harris county flood warning system, http://www.harriscountyfws.org/

[3] National seismological center, nepal, http://www .seismonepal.gov.np/

[4] Caltrans performance measurement system (pems), http://pems.dot.ca. gov/.

[5] D. Wang, L. Kaplan, H. Le, T. Abdelzaher, On truth discovery in social sensing: A maximum likelihood estimation approach in: Proceedings of the 11th International Conference on Information Processing in Sensor Networks, IPSN '12, ACM, New York, NY, USA, 2012, pp. 233-244. doi:10.1145/2185677. 2185737

URL http://doi.acm.org/10.1145/2185677.2185737

[6] K. Lee, R. Ganti, M. Srivatsa, P. Mohapatra, Spatio-temporal provenance: Identifying location information from unstructured text, IQ2S - Workshop at PerCom, 2013.

[7] P. Giridhar, M. T. Amin, T. F. Abdelzaher, L. M. Kaplan, J. George, R. K. Ganti, Clarisense: Clarifying sensor anomalies using social network feeds, in: 2014 IEEE International Conference on Pervasive Computing and Communication Workshops, PerCom 2014 Workshops, Budapest, Hungary, March 24-28, 2014, 2014, pp. 395-400. doi:10.1109/PerComW.2014.6815239

URL http://dx.doi.org/10.1109/PerComW.2014.6815239

[8] V. Chandola, A. Banerjee, V. Kumar, Anomaly detection: A survey, ACM Comput. Surv. 41 (3) (2009) 15:1-15:58. doi:10.1145/1541880.1541882. URL http://doi.acm.org/10.1145/1541880.1541882

[9] A. Lazarevic, A. Ozgur, L. Ertoz, J. Srivastava, V. Kumar, A comparative study of anomaly detection schemes in network intrusion detection, in: In Proceedings of the Third SIAM International Conference on Data Mining, 2003.

[10] J. Douceur, J. S. Donath, The sybil attack, 2002, pp. 251-260.

[11] D. Engler, D. Y. Chen, S. Hallem, A. Chou, B. Chelf, Bugs as deviant behavior: A general approach to inferring errors in systems code, in: Proceedings of the Eighteenth ACM Symposium on Operating Systems Principles, SOSP '01, ACM, New York, NY, USA, 2001, pp. 57-72. doi:10.1145/502034.502041. URL http://doi .acm.org/10.1145/502034.502041

[12] P. Mohan, V. N. Padmanabhan, R. Ramjee., Nericell: using mobile smartphones for rich monitoring of road and traffic conditions., In Proc. of ACM Conference on Embedded Networked Sensor Systems (SenSys), 2008. 
[13] R. Ganti, N. Pham, H. Ahmadi, S. Nangia, T. Abdelzaher., Greengps: A participatory sensing fuel-efficient maps application., In Proceedings of ACM 8th Annual International Conference on Mobile Systems, Applications and Services (MobiSys), 2010.

[14] H. Becker, M. Naaman, L. Gravano, Beyond trending topics: Real-world event identification on twitter.

[15] J. Weng, B.-S. Lee, Event detection in twitter URL http://www.aaai.org/ocs/index.php/ICWSM/ICWSM11/paper/ view/2767

[16] M. Paul, M. Dredze, You are what you tweet: Analyzing twitter for public health (2011).

URL http://www.aaai.org/ocs/index.php/ICWSM/ICWSM11/paper/ view/2880

[17] M.-D. Albakour, C. Macdonald, I. Ounis, Identifying local events by using microblogs as social sensors in: Proceedings of the 10th Conference on Open Research Areas in Information Retrieval, OAIR '13, Paris, France, France, 2013, pp. 173-180.

URL http://dl.acm.org/citation. cfm?id=2491748.2491785

[18] B. Pan, Y. Zheng, D. Wilkie, C. Shahabi, Crowd sensing of traffic anomalies based on human mobility and social media, in: ACM SIGSPATIAL GIS 2013, ACM, 2013.

URL http://research.microsoft.com/apps/pubs/default.aspx?id= 201131

[19] P. Giridhar, T. Abdelzaher, J. George, L. Kaplan, On quality of event localization from social network feeds, in: Pervasive Computing and Communication Workshops (PerCom Workshops), 2015 IEEE International Conference on, 2015, pp. 75-80. doi:10.1109/PERCOMW. 2015.7133997.

[20] Open baltimore data, https://data.baltimorecity.gov/Public-Safety/ Crime/e4ud-pvfr

[21] R. Balakrishnan, S. Kambhampati, Sourcerank: Relevance and trust assessment for deep web sources based on inter-source agreement, in: Proceedings of the 19th International Conference on World Wide Web, WWW '10, ACM, New York, NY, USA, 2010, pp. 1055-1056. doi:10.1145/1772690.1772801 URL http://doi.acm.org/10.1145/1772690.1772801

[22] X. Yin, W. Tan, Semi-supervised truth discovery, in: Proceedings of the 20th International Conference on World Wide Web, WWW '11, ACM, New York, NY, USA, 2011, pp. 217-226. doi:10.1145/1963405.1963439.

URL http://doi .acm.org/10.1145/1963405.1963439 